\title{
Emerging Adulthood: What Is It, and What Is It Good For?
}

\author{
Jeffrey Jensen Arnett \\ Clark University
}

ABSTRACT-This article asserts that the theory of emerging adulthood is a useful way of conceptualizing the lives of people from their late teens to their mid-to late $20 \mathrm{~s}$ in industrialized societies. The place of emerging adulthood within the adult life course is discussed. The weaknesses of previous terms for this age period are examined, and emerging adulthood is argued to be preferable as a new term for a new phenomenon. With respect to the question of whether emerging adulthood is experienced positively or negatively by most people, it is argued that it is positive for most people but entails developmental challenges that may be difficult and there is great heterogeneity, with some emerging adults experiencing serious problems. With respect to the question of whether or not emerging adulthood is good for society, it is argued that claims of the dangers of emerging adulthood are overblonon, but emerging adulthood is probably a mixed blessing for society.

KEYWORDS_emerging adulthood; young adulthood; transition to adulthood

It is now 7 years since I first proposed the term emerging adulthood for the age period from the late teens through the midto late 20s (roughly ages 18-25) in an article in American Psychologist (Arnett, 2000). I had mentioned the term briefly in two previous articles (Arnett, 1998; Arnett \& Taber, 1994), but the 2000 article was the first time I presented an outline of the theory. It was not until 2004 that I proposed a full theory in a book on emerging adulthood (Arnett, 2004). In a short time, the theory has become widely used, not just in psychology but in many fields. At the recent Third Conference on Emerging Adulthood (see www.ssea.org), a remarkable range of disciplines was represented, including psychology, psychiatry,

Correspondence concerning this article should be addressed to Jeffrey Jensen Arnett, Department of Psychology, Clark University, 950 Main Street, Worcester, MA 01602; e-mail: arnett@ jeffreyarnett.com. sociology, anthropology, education, epidemiology, health sciences, human development, geography, nursing, social work, philosophy, pediatrics, family studies, journalism, and law.

The swift spread of the term and the idea has surprised me because normally any new theoretical idea meets initial resistance from defenders of the reigning paradigm. Perhaps, the acceptance of emerging adulthood has been so swift because there really was no reigning paradigm. Instead, there was a widespread sense among scholars interested in this age period that previous ways of thinking about it no longer worked and there was a hunger for a new conceptualization. In any case, now that emerging adulthood has become established as a way of thinking about the age period from the late teens through at least the mid-20s, the theory is attracting commentary and critiques (e.g., Bynner, 2005). This is a normal and healthy part of the development of any new theory, and I welcome the exchange here with Leo Hendry and Marion Kloep.

\section{THE CONFIGURATION OF EMERGING ADULTHOOD: HOW DOES IT FIT INTO THE LIFE COURSE?}

When I first proposed the theory of emerging adulthood (Arnett, 2000 ), one of my goals was to draw attention to the age period from the late teens through the mid-20s as a new period of the life course in industrialized societies, with distinctive developmental characteristics. The dominant theory of the life course in developmental psychology, first proposed by Erikson (1950), postulated that adolescence, lasting from the beginning of puberty until the late teens, was followed by young adulthood, lasting from the late teens to about age 40 when middle adulthood began. This paradigm may have made sense in the middle of the 20th century when most people in industrialized societies married and entered stable full-time work by around age 20 or shortly after. However, by the end of the century, this paradigm no longer fit the normative pattern in industrialized societies. Median ages of marriage had risen into the late $20 \mathrm{~s}$, and the early to mid-20s became a time of frequent job changes 
and, for many people, pursuit of postsecondary education or training. Furthermore, sexual mores had changed dramatically, and premarital sex and cohabitation in the 20 s had become widely accepted. Most young people now spent the period from their late teens to their mid-20s not settling into long-term adult roles but trying out different experiences and gradually making their way toward enduring choices in love and work.

The theory of emerging adulthood was proposed as a framework for recognizing that the transition to adulthood was now long enough that it constituted not merely a transition but a separate period of the life course. I proposed five features that make emerging adulthood distinct: it is the age of identity explorations, the age of instability, the self-focused age, the age of feeling in-between, and the age of possibilities (Arnett, 2004). But I emphasized from the beginning that emerging adulthood is perhaps the most heterogeneous period of the life course because it is the least structured, and the five features were not proposed as universal features but as features that are more common during emerging adulthood than in other periods.

In this light, of the possible configurations A-D in Figure 1 of how emerging adulthood might fit into the adult life course, I would reject $\mathrm{D}$ because it does not show a distinct period between adolescence and adulthood. $\mathrm{C}$ does not work because it slights emerging adulthood, inaccurately portraying it as a brief transition between adolescence and adulthood. A is better, but it shows the transitions from adolescence to emerging adulthood and from emerging adulthood to young adulthood as more discrete than they actually are in some respects. It applies to transitions from adolescence to emerging adulthood such as finishing secondary school and reaching the legal age of adult status, and perhaps to transitions from emerging to young adulthood such as marriage. However, B works best in my view

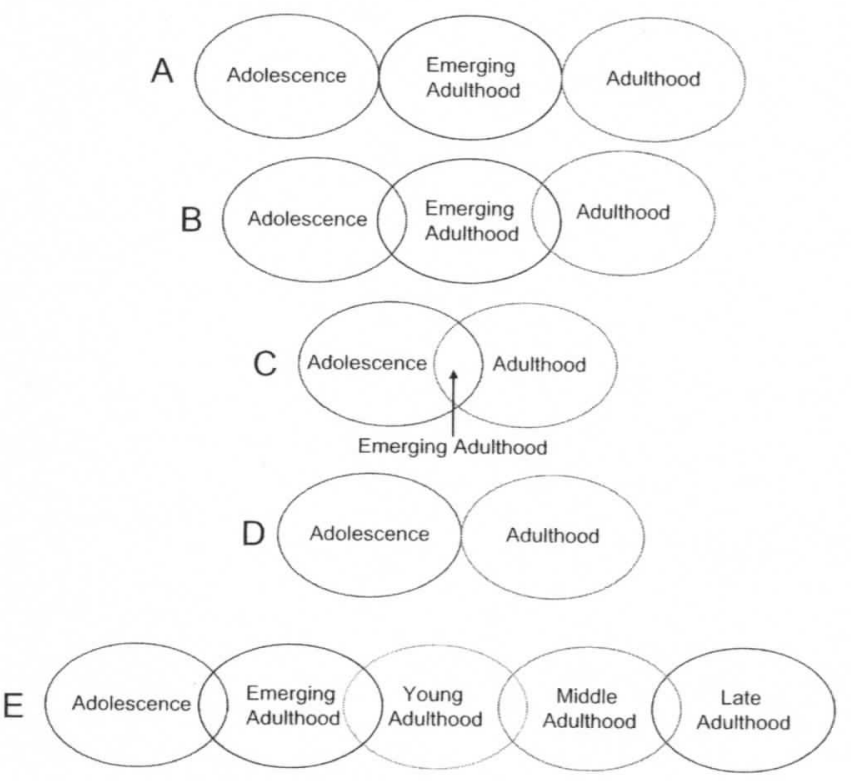

Figure 1. Possible configurations of emerging adulthood. because the five features described above are entered and exited not discretely but gradually. Furthermore, of the three criteria found in many countries and cultures to be the most important markers of reaching adult status - accepting responsibility for oneself, making independent decisions, and becoming financially independent - all are attained gradually in the course of emerging adulthood (Amett, 2001, 2003; Facio \& Micocci, 2003; Macek, Bejček, \& Vaníčková, 2007; Mayseless \& Scharf, 2003; Nelson, Badger, \& Wu, 2004).

This gradual passage from one period to the next may apply not just to emerging adulthood but to the entire adult life course. Theorists have emphasized how in recent decades the life course in industrialized societies has become increasingly characterized by individualization, meaning that institutional constraints and supports have become less powerful and important and people are increasingly left to their own resources in making their way from one part of the life course to the next, for better or worse (e.g., Côté, 2000; Heinz, 2002). Emerging adulthood is one part of this trend. So, in Figure 1, an improvement on $\mathrm{B}$ might be $\mathrm{E}$, showing gradual transitions into and out of different periods throughout the adult life course.

\section{DO WE REALLY NEED THE TERM EMERGING ADULTHOOD?}

I believe the rapid spread of the term emerging adulthood reflects its usefulness and the dissatisfaction of scholars in many fields with the previous terms that had been used. There were problems with each of those terms, including late adolescence, young adulthood, the transition to adulthood, and youth (Arnett, 2004). Late adolescence does not work because the lives of persons in their late teens and $20 \mathrm{~s}$ are vastly different from the lives of most adolescents (roughly ages 10-17). Unlike adolescents, 18- to 25-year-olds are not going through puberty, are not in secondary school, are not legally defined as children or juveniles, and often have moved out of their parents' household. Young adulthood does not work because it has been used already to refer to such diverse age periods, from preteens ("young adult" books) to age 40 ("young adult" social organizations). Furthermore, if 18-25 are "young adulthood," what are people who are 30,35 , or 40 ? It makes more sense to reserve "young adulthood" for the age period from about age 30 to about age 40 (or perhaps 45) because by age 30 most people in industrialized societies have settled into the roles usually associated with adulthood: stable work, marriage or other long-term partnership, and parenthood.

The transition to adulthood has been widely used in sociology and in research focusing mostly on the timing and sequence of transition events such as leaving home, finishing education, marriage, and parenthood. Certainly, the years from the late teens through the 20 s are when the transition to adulthood takes place for most people, not only as defined by transition events but also by a more subjective sense of having reached adulthood 
(Arnett, 1998, 2001, 2003). But why call this period merely a "transition" rather than a period of development in its own right? If we state, conservatively, that it lasts 7 years, from age 18 to 25 , that makes it longer than infancy, longer than early or middle childhood, and as long as adolescence. Furthermore, calling it "the transition to adulthood" focuses attention on the transition events that take place mainly at the beginning or end of the age range, whereas calling it "emerging adulthood" broadens the scope of attention to the whole range of areascognitive development, family relationships, friendships, romantic relationships, media use, and so on-that apply to other developmental periods as well.

Finally, youth has been used as a term for this period, especially in Europe but also among some American psychologists and sociologists. However, youth suffers from the same problem as young adulthood, in that it has long been used to refer to a wide range of ages, from middle childhood ("youth organizations") through the 30 s. Furthermore, in its American incarnation, it was promoted by Keniston (1971) on the basis of his research with student protesters in the late $1960 \mathrm{~s}$, and his description of it as a time of rebellion against society bears the marks of his time but does not apply widely.

Emerging adulthood is preferable because it is a new term for a new phenomenon. Across industrialized societies in the past half century, common changes have taken place with respect to the lives of young people: longer and more widespread participation in postsecondary education and training, greater tolerance of premarital sex and cohabitation, and later ages of entering marriage and parenthood. As a consequence of these changes, a new period of the life course has developed between adolescence and young adulthood. Furthermore, emerging adulthood reflects the sense among many people in the late teens and early 20 s worldwide that they are no longer adolescent but only partly adult, emerging into adulthood but not there yet (e.g., Arnett, 2003; Macek et al., 2007; Mayseless \& Scharf, 2003; Nelson et al., 2004).

Some aspects of the theory of emerging adulthood are likely to be modified with further research, and the main features of emerging adulthood will no doubt vary among cultures. There are certainly psychosocial differences among emerging adults related to socioeconomic status and ethnic group, and crossnational differences have only begun to be explored (e.g., Buhl, 2007; Facio \& Micocci, 2003; Fadjukoff, Kokko, \& Pulkinnen, 2007; Lanz \& Tagliabue, 2007). But there is some degree of heterogeneity in every developmental period, and overarching terms and general descriptions for those periods are nevertheless useful for understanding them (Arnett, 2006b).

\section{IS EMERGING ADULTHOOD EXPERIENCED POSITIVELY OR NEGATIVELY BY MOST PEOPLE?}

The fact that it takes longer to reach full adulthood today than it did in the past has been subject to various interpretations, mostly negative. In American popular media, the term "quarterlife crisis" has been coined to describe the alleged difficulties experienced by emerging adults as they try to find a place in the adult world (Robbins \& Wilner, 2001). Within academia, some sociologists have asserted that higher ages of marriage and parenthood indicate that "growing up is harder to do" than in the past.

Yet, the bulk of the evidence is contrary to these assertions (Arnett, 2007b). Numerous studies show that for most, wellbeing improves during the course of emerging adulthood. An example is shown in Figures 2 and 3, which demonstrate a decline in depressive symptoms and a rise in self-esteem in a longitudinal Canadian study of emerging adults (Galambos, Barker, \& Krahn, 2006). Similar results have been found in the longitudinal Monitoring the Future studies in the United States (Schulenberg \& Zarrett, 2006). Emerging adults enjoy their selffocused freedom from role obligations and restraints, and they take satisfaction in their progress toward self-sufficiency. I think they also benefit from growing social cognitive maturity, which enables them to understand themselves and others better than they did as adolescents (Arnett, 2004).

Nevertheless, although I believe the notion of a "quarterlife crisis" is exaggerated, I do not dismiss it entirely. It is true that identity issues are prominent in emerging adulthood and that sorting through them and finding satisfying alternatives in love and work can generate anxiety. The idea of a "quarterlife crisis" can be seen as recognizing that the identity crisis

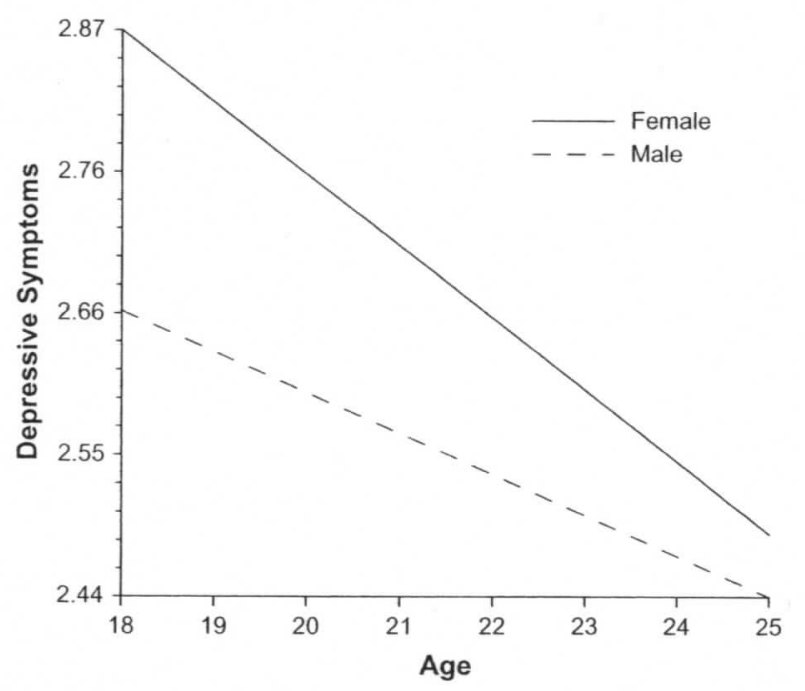

Figure 2. Depressive symptoms decline during emerging adulthood. Note. The sample was drawn from Grade 12 classes in six high schools in a western Canadian city, followed over the next 7 years. $N=920$ at age 18 and 324 at age 25. The sample was diverse in socioeconomic status background; at Time 1, 10\% were from families in which both parents had a university degree, and $16 \%$ had one parent with a university degree. Among the participants themselves, by age $25,30 \%$ had a university degree, $14 \%$ had a college diploma, $24 \%$ had a technical degree, and the remaining $32 \%$ had no postsecondary educational credential.

Source: Galambos, Barker, and Krahn (2006). 
Figure 3. Self-esteem rises during emerging adulthood.

Note. The sample was drawn from Grade 12 classes in six high schools in a western Canadian city, followed over the next 7 years. $N=920$ at age 18 and 324 at age 25 . The sample was diverse in socioeconomic status background; at Time 1, 10\% were from families in which both parents had a university degree, and $16 \%$ had one parent with a university degree. Among the participants themselves, by age $25,30 \%$ had a university degree, $14 \%$ had a college diploma, $24 \%$ had a technical degree, and the remaining $32 \%$ had no postsecondary edueational credential. Source: Galambos, Barker, and Krahn (2006).

Erikson (1950) described over a half century ago as central to adolescence has now moved into emerging adulthood. It is also true that entry into the labor market is often stressful and frustrating, especially for emerging adults with limited educational credentials (Côté, 2000; Hamilton \& Hamilton, 2006). Furthermore, even among the most advantaged emerging adults, the graduates of 4-year colleges and universities, their extraordinarily high expectations for the workplace - their aspirations of finding work that not only pays well but also provides a satisfying and enjoyable identity fit - are difficult for reality to match and often require compromises of their hopes and dreams (Arnett, 2004). Nevertheless, the evidence of rising well-being during the course of emerging adulthood indicates that most people adapt successfully to its developmental challenges.

Here as elsewhere, we must take into account the heterogeneity of emerging adults. Even as well-being rises for most emerging adults, some experience serious mental health problems such as major depression and substance use disorder (Schulenberg \& Zarrett, 2006; Tanner, Arnett, \& Leis, in press; Tanner et al., 2007). A possible interpretation is that the variance in mental health functioning becomes broader in the course of emerging adulthood (Arnett, 2007a). This may be because emerging adults have fewer social roles and obligations than children and adolescents, whose lives are structured by their parents and other adults, or adults (beyond emerging adulthood), whose lives are structured by work, family, and community roles and obligations. Although most emerging adults appear to thrive on this freedom, some find themselves lost and may begin to experience serious mental health problems. Emerging adults may also struggle if they are part of especially vulnerable populations, such as those aging out of foster care, coming out of the criminal justice system, or experiencing disabilities (Osgood, Foster, Flanagan, \& Ruth, 2005).

Although I have made a case that emerging adulthood is experienced positively by most people, I hasten to add that my perspective is based mainly on my interviews and other data obtained from emerging adults in the United States and (recently) Denmark. Studies on emerging adults in other countries, such as Argentina (Facio \& Micocci, 2003), Israel (Mayseless \& Scharf, 2003), Czech Republic (Macek et al., 2007), and China (Nelson et al., 2004) show some similarities as well as some differences. An exciting prospect for the new field of emerging adulthood is examining the forms it takes in different countries and cultures worldwide (Arnett, 2006c). The articles in this Special Section are an important step toward this goal.

\section{IS EMERGING ADULTHOOD GOOD FOR SOCIETY?}

Even if it is true that most people seem to enjoy their emerging adulthood, is the advent of this new period of life good for society? Certainly, there are complaints about it in American popular media. "They Just Won't Grow Up" sniggered a TIME magazine cover story on emerging adults in 2005. In the 2006 movie Failure to Launch, a young man shows so little inclination to take on adult responsibilities that his parents hire an attractive young woman to lure him out of their household. Advice writers warn that emerging adults are refusing to give up their teenage pleasures and take on adult responsibilities, with "catastrophic" results (Levine, 2005, p. 19).

Here, as with "quarterlife crisis," a grain of truth is exaggerated to the point of caricature (Arnett, 2007a). It is true that many emerging adults are ambivalent about taking on adult roles and responsibilities (Arnett, 2004). Although they take a certain satisfaction in moving toward self-sufficiency, they also find it burdensome and onerous to pay their own bills and do all the other things their parents had always done for them. Furthermore, they often view adulthood as dull and stagnant, the end of spontaneity, the end of a sense that anything is possible.

Nevertheless, their ambivalence is not an outright refusal or rejection of adult roles. It may be that they are wise to recognize the potentials of emerging adulthood and to wait until at least their late 20 s to take on the full range of adult obligations. Although adulthood may have more satisfactions and rewards than they recognize, they are right that entering adult roles of marriage, parenthood, and stable full-time work entails constraints and limitations that do not apply in emerging 
adulthood. Once adult roles are entered they tend to be enduring if not lifelong. It seems sensible for emerging adults to wait to enter them until they judge themselves to be ready, and meanwhile to enjoy the freedoms of emerging adulthood while they last.

It should also be added that few emerging adults fail to "grow up" and take on the responsibilities of adulthood. By age 30, three fourths of Americans are married, three fourths have at least one child, nearly all have entered stable employment, nearly all have become financially independent from their parents, and almost none live in their parents' household (Arnett, 2004; Goldscheider \& Goldscheider, 1999). Similarly, by age 30 nearly all (about 90\%) feel that they have fully reached adulthood, no longer feeling in-between (Arnett, 2001). Thus, the claim that a long and gradual process of taking on adult responsibilities during emerging adulthood results in permanent rejection of adulthood is clearly overblown. The great majority of emerging adults become contributing young adult members of society by age 30 , fulfilling stable family and work roles.

Here again, my perspective is based mainly on my research with American emerging adults. However, there are some indications that similar patterns exist in most other industrialized countries, with some variations. Across industrialized societies emerging adulthood is a period of many changes in love and work but most people settle into enduring adult roles by about age 30 (Arnett, 2006a; Iacovou, 2002; Sneeding \& Phillips, 2002).

So, emerging adulthood may not be harmful to societies, but is it actually good for them? Yes and no. On the one hand, it would be nice to think that if people spend most of their $20 \mathrm{~s}$ looking for just the right job and just the right love partner, they will have a better chance of finding happiness in love and work than if they had made long-term commitments in their late teens or very early 20 s out of duty, necessity, or social pressure. On the other hand, emerging adults' expectations for love and work tend to be extremely high-not just a reliable marriage partner but a "soul mate," not just a steady job but a kind of work that is an enjoyable expression of their identity - and if happiness is measured by the distance between what we expect out of life and what we get, emerging adults' high expectations will be difficult for real life to match. So, it cannot be said with confidence that the existence of emerging adulthood ensures that most people in a society will be happier with their adult lives.

Furthermore, emerging adulthood is the peak age period for many behaviors most societies try to discourage, such as binge drinking, illegal drug use, and risky sexual behavior (Arnett, 2000, 2005; Schulenberg \& Zarrett, 2006). If people still entered adult commitments around age 20 , as they did in the past, rates of risk behaviors in the 20 s would undoubtedly be lower. Such behavior may be fun for emerging adults, but it can hardly be said to be good for their society. However, one way emerging adulthood is good for society is that it allows young people an extended period that can be used for postsecondary education and training that prepares them to contribute to an information and technology-based global economy.

\section{CONCLUSION}

Already in its short life, emerging adulthood has been shown to bear the marks of a good theory: It has generated research, ideas, and critiques that have advanced science and scholarship. Like all theories, it is an imperfect model of real life, and will no doubt be subject to alterations, revisions, and elaborations in the years to come. Especially important will be investigating the different forms it takes in cultures around the world. The theory of emerging adulthood that I have presented is offered as a starting point, and I look forward to the contributions and further advances to come, from scholars around the world.

\section{REFERENCES}

Arnett, J. J. (1998). Learning to stand alone: The contemporary American transition to adulthood in cultural and historical context. Human Development, 41, 295-315.

Arnett, J. J. (2000). Emerging adulthood: A theory of development from the late teens through the twenties. American Psychologist, $55,469-480$.

Arnett, J. J. (2001). Conceptions of the transition to adulthood: Perspectives from adolescence to midlife. Journal of Adult Development, 8, 133-143.

Arnett, J. J. (2003). Conceptions of the transition to adulthood among emerging adults in American ethnic groups. New Directions in Child and Adolescent Development, 100, 63-75.

Arnett, J. J. (2004). Emerging adulthood: The winding road from the late teens through the twenties. New York: Oxford University Press.

Arnett, J. J. (2005). The developmental context of substance use in emerging adulthood. Journal of Drug Issues, 35, 235-253.

Arnett, J. J. (2006a). The case for emerging adulthood in Europe: A response to Brunner. Journal of Youth Studies, 9, 111-123.

Arnett, J. J. (2006b). Emerging adulthood: Understanding the new way of coming of age. In J. J. Arnett \& J. L. Tanner (Eds.), Emerging adults in America: Coming of age in the 21st century (pp. 3-19). Washington, DC: APA Books.

Arnett, J. J. (2006c). The psychology of emerging adulthood: What is known, and what remains to be known? In J. J. Arnett \& J. L. Tanner (Eds.), Emerging adults in America: Coming of age in the 21st century (pp. 303-330). Washington, DC: APA Book.

Arnett, J. J. (2007a). Socialization in emerging adulthood: From the family to the wider world, from socialization to self-socialization In J. Grusec \& P. Hastings (Eds.), Handbook of socialization (pp. 208-231). New York: Guilford.

Arnett, J. J. (2007b). Suffering, selfish, slackers? Myth and reality on emerging adults. Journal of Youth and Adolescence, 36, 23-29.

Arnett, J., \& Taber, S. (1994). Adolescence terminable and interminable: When does adolescence end? Journal of Youth and Adolescence, 23, 517-537. 
Levine, M. (2005). Ready or not, here life comes. New York: Simon \& Schuster.

Macek, P., Bejček, J., \& Vaníčková, J. (2007). Contemporary Czech emerging adults: Generation growing up in the period of social changes. Journal of Adolescent Research, 22, 444-475.

Mayseless, 0., \& Scharf, M. (2003). What does it mean to be an adult? The Israeli experience. New Directions in Child and Adolescent Development, 100, 5-20.

Nelson, L. J., Badger, S., \& Wu, B. (2004). The influence of culture in emerging adulthood: Perspectives of Chinese college students. International Journal of Behavioral Development, 28, $26-36$.

Osgood, D. W., Foster, E. M., Flanagan, C., \& Ruth, G. R. (2005). On your own without a net: The transition to adulthood for vulnerable populations. Chicago: University of Chicago Press.

Robbins, A., \& Wilner, A. (2001). Quarterlife crisis: The unique challenges of life in your twenties. New York: Tarcher/Putnam. emerging adulthood: Continuity and discontinuity in courses, causes, and functions. In J. J. Arnett \& J. L. Tanner (Eds.), Emerging adults in America: Coming of age in the 21st century (pp. 135-172). Washington, DC: APA Books.

Sneeding, T. M., \& Phillips, K. R. (2002). Cross-national differences in employment and economic sufficiency. Annals of the American Academy of Political Social Science, 580, 103-133.

Tanner, J. L., Arnett, J. J., \& Leis, J. A. (in press). Emerging adulthood: Learning and development during the first decade of adulthood. In M. C. Smith \& T. G. Reio (Eds.), Handbook of research on adult development and learning. Mahwah, NJ: Erlbaum.

Tanner, J. L., Reinherz, H. Z., Beardslee, W. R., Fitzmaurice, G. M., Leis, J. A., \& Berger, S. R. (2007). Change in prevalence of psychiatric disorders from ages 21 to 30 in a community sample. Journal of Nervous and Mental Disease, 195, 298-306.
Schulenberg, J. E., \& Zarrett, N. R. (2006). Mental health during Journal of Adolescent Research, 22, 531-549. 